\title{
On the heat transport in a sunspot penumbra
}

\author{
R. Schlichenmaier ${ }^{1}$ and S. K. Solanki ${ }^{2}$ \\ 1 Kiepenheuer-Institut für Sonnenphysik, Schöneckstr. 6, 79104 Freiburg, Germany \\ 2 Max-Planck-Institut für Aeronomie, Max-Planck-Str. 2, 37191 Katlenburg-Lindau, Germany \\ Received 23 April 2003 / Accepted 15 July 2003

\begin{abstract}
The penumbra radiates an energy flux that is roughly $75 \%$ of the quiet-sun value. One mechanism proposed to bring this flux to the surface is interchange convection of magnetic flux tubes according to which hot flux tubes rise to the surface, cool off their heat by radiation and sink down again. Another way to deposit heat in the penumbral photosphere is by steady upflows along magnetic flux tubes. We discuss these two mechanisms and elaborate on consequences that can be compared with and constrained by observations. We estimate the time scales for variations of the intensity and the magnetic field pattern. By comparing them with the corresponding observed time scales, we find that pure interchange convection is unable to account for the observed penumbral heat flux. Heating the penumbra by steady upflows along magnetic flux tubes, however, turns out the penumbra. Associated with the magnetic return flux, downflows within the penumbra should be present, in accordance with recent observational findings of such downflows. Exploring other possible heating mechanisms, we find that dissipation of magnetic energy is negligible, while dissipation of the kinetic energy of the Evershed flow could contribute significantly to the
\end{abstract} \\ to be sufficient to explain the penumbral brightness, under the condition that significant magnetic return flux is present within \\ brightness of the penumbra.
}

Key words. Sun: magnetic fields - Sun: photosphere - sunspots - magnetohydrodynamics

\section{Introduction}

The photospheric appearance of sunspots has been studied in considerable detail. They consist of the umbra and the penumbra, which appear on average darker than the surrounding granulation. Sunspots manifest concentrated magnetic fields with a strength of some 3000 Gauss at the center. The field strength gradually decreases outwards. At the outer sunspot boundary on the solar surface (continuum optical depth $\tau=1$ ), it reaches 800 Gauss and vanishes beyond.

Both the umbra and the penumbra show fine structure at the photospheric level (e.g., Sobotka 1997; Solanki 2003; Scharmer et al. 2002). In the umbra, "umbral dots" are observed, while in the penumbra the fine structure is commonly referred to as "penumbral grains" or "penumbral filaments". Moreover, the fine structure seen in intensity images of the penumbra is accompanied by a predominantly horizontal flow, the Evershed flow, and by fluctuations of the magnetic field vector on small spatial scales.

The morphology and the structure of sunspots beneath the photosphere is still unclear in detail, mainly because of our lack in understanding the relevant magnetoconvective processes (see reviews of Weiss 1997, 2002; Jahn 1997; Hurlburt et al. 2000; Solanki 2001). Simulations of magnetoconvection in an inclined magnetic field by Hurlburt et al. (1996) mimicking the penumbra suggest that overturning convection is modulated by the inclined magnetic field such that the convection

Send offprint requests to: R. Schlichenmaier, e-mail: schliche@kis.uni-freiburg.de cells become elongated and inclined. Under certain conditions, these cells can migrate in a direction opposite to the tilt of the field, leading to the following picture: intensity waves migrate inwards toward the umbra, while a net velocity at the surface is directed in the direction of the field tilt, i.e., outwards. This study demonstrates that magnetoconvective solutions are conceivable that are in qualitative agreement with the observed migration of penumbral grains and the observed (Evershed) outflow. But this study cannot be used to quantitatively describe the energy transport in penumbrae, because the parameters and boundary conditions used in this simulation are far from those appropriate for the Sun.

Magneto-hydrostatic sunspot models (e.g., Pizzo 1986, 1990; Pizzo et al. 1993; Jahn 1989; Jahn \& Schmidt 1994) are capable of properly describing the global properties of sunspots. Yet, in these models the magneto-convective heat transport is parameterized such that the observed surface temperatures are reproduced. In such monolithic models, the sunspot is confined by a magnetopause and harbours a penumbra, in which the magnetic field is inclined with respect to the vertical. In particular, they consist of a deep penumbra, i.e. the penumbra extends many scale heights beneath the photosphere. The fact that the penumbra should be deep rather than shallow was demonstrated by Schmidt (1991) and Solanki \& Schmidt (1993). As a consequence the energy in the penumbra cannot be transported by convective rolls extending one scale height as proposed by Danielson (1961). Rather a magneto-convective transport of enthalpy acting over many scale heights must be present. 
A possible scenario for the heat transport in sunspot penumbrae has been proposed by Jahn \& Schmidt (1994), namely the interchange convection of magnetic flux tubes: Flux tubes that lie along the magnetopause become heated by the hotter field-free surroundings and start to rise by interchanging positions with other flux tubes until they reach the photosphere, where they form the observed bright penumbral filaments. Then, they deposit their surplus heat by radiation, and sink back to the magnetopause, where they heat up again.

Investigating the interchange scenario, Schlichenmaier et al. (1998a,b) modelled the dynamics of a thin magnetic flux tube that initially lies along the magnetopause. They found that the tube is indeed heated up by the field-free hotter gas, in layers just beneath the photosphere, such that the tube becomes buoyant and rises to the surface. However, in those calculations an upward flow along the tube develops and the inertia of this flow prevents the tube from sinking back down. The footpoint of the tube migrates inwards (towards the umbra), and finally reaches a nearly steady state in which the tube continues to lie horizontally in the photosphere with a bright footpoint harbouring an upflow in the inner penumbra and an associated outflow in the horizontal part of the tube. This model reproduces a significant number of observational aspects (Schlichenmaier 1999).

Hence, these calculations suggest that interchange convection as originally proposed does not take place in the penumbra of a sunspot. In this paper, we elaborate on the question about the heat transport by estimating the amount of heat that could be transported by interchange convection (Sect. 2), and the amount of heat that is deposited by a steady upflow in the penumbral photosphere (Sect. 3). We infer observational constraints which are compared to MDI observations in which the temporal and spatial fluctuations of the intensity and the magnetic field are investigated. These observations have only a moderate spatial resolution ( $0.6^{\prime \prime}$ per pixel), but the advantage of being unaffected by seeing fluctuations of the earth's atmosphere. The results of the data analysis are given by Solanki \& Rüedi (2003, hereafter Paper I). We argue that interchange convection seems unlikely, because one would expect fluctuations of the intensity and the magnetic field pattern on a much smaller time scale than seen in the observations. Steady upflows as the relevant heating mechanism are supported by the MDI observations, but downflows within the penumbra are needed to allow for sufficiently many upflow channels that are necessary to explain the penumbral brightness. We discuss consequences of our findings and mention other heating processes that may contribute to the brightness of the penumbra in Sect. 3.1. Our conclusions are presented in Sect. 4.

\section{Interchange convection}

\subsection{Cooling time versus time scale of intensity variation}

In this section we consider a freshly emerged, hot flux tube and compare its radiative cooling time scale with the observed time scale of penumbral intensity variations.
The simulations of the moving tube model by Schlichenmaier et al. (1998a,b) suggest that a rising tube enters the photosphere with $T=12000 \mathrm{~K}$ at a density of $\rho=2 \times 10^{-7} \mathrm{~g} \mathrm{~cm}^{-3}$. Schlichenmaier et al. (1999, hereafter SBS1999) have investigated the cooling behaviour for such a hot, horizontal flux tube embedded in a gas similar to the dark penumbral background, i.e. the dark fibrils. A tube at $12000 \mathrm{~K}$ is optically thick because the $\mathrm{H}^{-}$opacity is extremely temperature sensitive. Gas at this high temperature is not seen, since the surrounding plasma is radiatively heated such that the continuum optical depth lies outside the tube proper, being located at a lower temperature $(\$ 7000 \mathrm{~K}$, cf. Sect. 7 in SBS1999). They determined the cooling time, $\tau_{\text {cool }}$ (i.e., the e-folding time of temperature difference between flux tube and surroundings), for flux tubes with radii $R$ ranging from 25 to $400 \mathrm{~km}$. Based on multi-dimensional radiative transfer calculations, they find that $\tau_{\text {cool }} \sim R^{1.5}$, with $\tau_{\text {cool }} \approx$ $30 \mathrm{~s}$ for $R=100 \mathrm{~km}$, and $\tau_{\text {cool }} \approx 250 \mathrm{~s}$ for $R=400 \mathrm{~km}$. That is, even a flux tube as thick as $800 \mathrm{~km}$, which would easily be resolved by MDI, has a radiative cooling time that is by far smaller than the observed time scale of intensity variation, which amounts to at least $30 \mathrm{~min}$ (cf. Table 1 of Paper I). Obviously, the cooling time critically depends on the temperature of the emerged tube, being longer for hotter tubes. For an extremely hot tube of $14400 \mathrm{~K}$ and radii of $100 \mathrm{~km}$ and $400 \mathrm{~km}$, SBS1999 find a cooling time of $1 \mathrm{~min}$ and $9 \mathrm{~min}$, respectively. Such a high temperature seems unrealistic, but even then the cooling time is considerably shorter than the time scale of intensity variation $(30 \mathrm{~min})$.

Already this suggests that, if it takes place, interchange convection must occur as a process in which flux tubes rise and sink continuously, which is not seen in the analysed MDI data set. Moreover, ground-based penumbral time series of better spatial resolution give average life times for the penumbral filaments (grains) that are longer than some $30 \mathrm{~min}$ (e.g., Sobotka $\&$ Sütterlin 2001). This means that bright penumbral grains live much longer than the time required for the emerged tubes to radiate off their surplus energy. Hence, the energy in the grains must be replenished continuously (if they are indeed due to emerging tubes).

\subsection{Effectiveness of interchange convection}

In this section we estimate the rate at which flux tubes with radius, $R$, have to keep emerging in order to acount for the brightness of the penumbra. First we estimate the amount of heat that is deposited per unit area (of $1 \mathrm{~cm}^{2}$ ) by an emerged flux tube. Assuming that it radiatively cools from $12000 \mathrm{~K}$ to approximately $5000 \mathrm{~K}$, it deposits $u=3 \times 10^{12} \mathrm{erg} \mathrm{g}^{-1}$. This value is obtained for a solar mixture of hydrogen, helium and metals while taking into account partial ionization of hydrogen and helium (cf. SBS1999). Since the heat is advected into the photosphere where it radiates away, the deposited energy fully contributes to the penumbral brightness.

The energy, $e$, deposited by a tube with radius, $R$, and length, $l$, amounts to $e=u \rho\left(\pi R^{2}\right) l$. Since the tube has a 
diameter (width) of $2 R$, its energy, $E$, deposited per unit area in the photosphere is given by $E=e /(2 R l)$, i.e.,

$E=u \rho \frac{R \pi}{2} \approx\left(\frac{R}{100 \mathrm{~km}}\right) \times 10^{13} \mathrm{erg} \mathrm{cm}^{-2}$,

for the appropriate values of $u$ and $\rho$.

The radiative heat flux from the quiet sun amounts to $F_{\odot}=6.3 \times 10^{10} \mathrm{ergs} \mathrm{s}^{-1} \mathrm{~cm}^{-2}$, i.e., $T_{\text {eff } \odot}=5778 \mathrm{~K}$ (Stix 2002). The umbral and penumbral radiative heat fluxes are given approximately by $F_{\mathrm{pu}}=0.75 F_{\odot}\left(T_{\mathrm{eff}}\right.$, pu $\left.=5375 \mathrm{~K}\right)$ and $F_{\mathrm{u}}=0.23 F_{\odot}\left(T_{\text {eff, u }}=4000 \mathrm{~K}\right)$, respectively (e.g., Thomas \& Weiss 1992).

The value of Eq. (1) is to be compared to the radiated penumbral heat flux, $F_{\mathrm{pu}}=5 \times 10^{10} \mathrm{erg} \mathrm{s}^{-1} \mathrm{~cm}^{-2}$. In order to supply this observed penumbral brightness, the time span between successive emergences has to be:

$\tau_{\text {emerge }}=\frac{E}{F_{\text {pen }}} \approx\left(\frac{R}{100 \mathrm{~km}}\right) 200 \mathrm{~s}$.

This time span corresponds to the time scale at which the magnetic pattern is expected to change. The emergence time scale is found to be larger than the cooling time scale, meaning that the tubes have sufficient time to radiate off their energy.

For an upper limit of $\tau_{\text {emerge}}$, we may assume that interchange convection only accounts for the surplus brightness, $F_{\mathrm{sb}}$, of the penumbra relative to the umbra,

$F_{\mathrm{sb}}=F_{\mathrm{pen}}-F_{\mathrm{u}} \approx 1 / 2 F_{\odot} \approx 3 \times 10^{10} \mathrm{erg} \mathrm{s}^{-1} \mathrm{~cm}^{-2}$.

This implies that some (unidentified) small-scale magnetoconvective process invoked to explain the brightness of the umbra is also present in the penumbra, and that we only need to explain the extra brightness of the penumbra relative to the umbra. Then the time span increases to $\tau_{\text {emerge }}=(R / 100 \mathrm{~km}) 300 \mathrm{~s}$. With the highest values that seem reasonable for the temperature of the emerging tube, $T=13000 \mathrm{~K}$ (which gives $u=4 \times 10^{12} \mathrm{erg} \mathrm{g}^{-1}$ ) and the density, $\rho=3 \times 10^{-7} \mathrm{~g} \mathrm{~cm}^{-3}$, one increases the heat content of the tube and obtains an upper limit for the time span of $\tau_{\text {emerge }}<(R / 100 \mathrm{~km}) 600 \mathrm{~s}$.

For the upper limit of a typical penumbral tube with a radius of $100 \mathrm{~km}, \tau_{\text {emerge }}=300 \mathrm{~s} \approx 5 \mathrm{~min}$. For a tube with $R=400 \mathrm{~km}, \tau_{\text {emerge }} \approx 20 \mathrm{~min}$. Even for such thick tubes, which could be resolved by MDI, the emergence time scale is significantly shorter than the observed time scale for the variation of the magnetic pattern, which is found to be at least 80 min (cf. Table 1 of Paper I). The upper limit of $\tau_{\text {emerge }}$ is also shorter than the time scale of intensity variation in the penumbra, which is at least $30 \mathrm{~min}$.

Hence, there are two problems with explaining the high brightness of penumbrae based on the original idea of interchange convection within the constraints set by MDI: (1) the radiative cooling time scale of such tubes is by an order of magnitude smaller than the time scale of intensity variations, and (2) the time span between successive emergences of tubes (which is needed to explain the brightness of the penumbra) is much smaller than the time scale at which the magnetic pattern changes.
One possibility compatible with the data are flux tubes much smaller than the pixel size. The cooling time then decreases even more and flux tubes need to emerge in even more rapid succession. Thus, a flux tube of, say, $R=1 \mathrm{~km}$ would have to be replaced every $4 \mathrm{~s}$ (such tubes have been proposed by Sánchez Almeida et al. 1996). The spatial scale of the variation would be so small that we cannot detect it. Observations, however, give evidence that bright penumbral structures are coherent elongated objects with a width of some $250 \mathrm{~km}$ (Sütterlin 2001), and a life time of more than $30 \mathrm{~min}$ (Sobotka \& Sütterlin 2001). We cannot exclude that a single penumbral filament is in reality a conglomerate of very small-scale objects. But it seems unlikely that those very small-scale objects are distributed uniformly throughout the penumbra, since then it is hard to explain the fact that penumbral filaments are seen at a width of $350 \mathrm{~km}$ having a life time that is much longer than the radiative cooling time that we estimate.

\section{Steady upflows along flux tubes}

The moving tube model of Schlichenmaier et al. (1998a,b) provides us with a possible alternative. It reveals a steady upflow of hot gas through the inner footpoint (i.e. the one closer to the umbra) of the emerged flux tube, which becomes a horizontal outflow along the horizontal section of the tube (and could explain the Evershed effect). As the gas flows upwards and outwards it radiates and thus cools. We now estimate the area that can be heated with such an upflow channel.

The upflow velocity at the footpoint returned by the model is $v=4 \mathrm{~km} \mathrm{~s}^{-1}$. Evidence for such upflows in the inner penumbra has been found (Rimmele 1995; Stanchfield et al. 1997; Westendorp Plaza et al. 1997; Schlichenmaier \& Schmidt 2000; Schmidt \& Schlichenmaier 2000; Schlichenmaier \& Collados 2002; Bellot Rubio et al. 2003). The heat, $F_{\text {upflow }}$, deposited in the photosphere by such an upflow per unit time and per unit area is:

$F_{\text {upflow }}=u v \rho \approx 24 \times 10^{10} \mathrm{erg} \mathrm{s}^{-1} \mathrm{~cm}^{-2} \approx 4 F_{\odot}$,

for typical values of $u, v$, and $\rho$.

If we assume that the upflows explain the surplus brightness of the penumbra, $F_{\mathrm{sb}}$ (cf. Eq. (3)), then an upper limit of the area that such an upflow channel of radius, $R$, can account for is given by:

$A=\frac{F_{\text {upflow }} \pi R^{2}}{F_{\mathrm{sb}}} \approx 25 R^{2}$.

Downstream of the upflow footpoint, the tube lies horizontally. Then, the area over which on average the penumbral flux is emitted (fed by a single flux tube footpoint) roughly equals that of a rectangle with a width that corresponds to the diameter of the tube and a length, $l$, of

$l=\frac{A}{2 R} \approx 12 R$.

That means that a tube with $R=100 \mathrm{~km}$ would heat a rectangular area with a length of $1200 \mathrm{~km}$, while for a tube with $R=200 \mathrm{~km}, l=2400 \mathrm{~km}$. 
This estimate indicates that upflows along flux tubes are in principle capable of accounting for the surplus brightness of the penumbra. However, another problem arises, due to a simple geometrical argument. According to the model the tube lies horizontally from the footpoint to the outer edge of the penumbra. But after a length $l$ from the footpoint, a new heat source (upflow channel) is needed to supply the surplus penumbral brightness of the next rectangular area. Yet, if the tube continues horizontally, there is no space for such a flow channel.

According to observations, the width of bright filaments (i.e., the diameter of the flow channel) should in any case not be larger than $400 \mathrm{~km}$, i.e., $R=200 \mathrm{~km}$ (e.g., Grossmann-Doerth \& Schmidt 1981; Sütterlin 2001). That means that $l$ is smaller than $2400 \mathrm{~km}$. Since typical penumbrae have a width that easily exceeds $5000 \mathrm{~km}$, and may exceed $10000 \mathrm{~km}$, a single flux tube is not capable of heating the entire width of a penumbra.

An increase of $l$ to heat the entire penumbral width could be achieved by increasing the temperature of the upflow, the density of the upflow, or by simply increasing the velocity of the upflow. On the basis of the numerical simulations of the moving tube model, a significant increase of the upflow velocity seems inconceivable. An extreme upper limit for the above estimate would therefore be obtained with $v=5 \mathrm{~km} \mathrm{~s}^{-1}, T=13000 \mathrm{~K}, \rho=3 \times 10^{-7} \mathrm{~g} \mathrm{~cm}^{-3}$. Even then, with $R=200 \mathrm{~km}$, we only obtain $l=25 R=5000 \mathrm{~km}$. From this we conclude that increasing the heat content of the upflow within reasonable limits does not suffice to heat the entire penumbral width with only one upflow channel.

Nevertheless, hot upflows would still be conceivable to account for the surplus brightness of the penumbra, if

(1) the horizontal flow channels are stacked vertically within the photosphere, i.e., lie at different heights;

or

(2) the flow downstream of the footpoint is not horizontal, but submerges beneath the photosphere while still within the penumbra.

Since the Evershed flow is observed to be located mainly in the deep photosphere (e.g., Maltby 1964; Schlichenmaier \& Schmidt 2000), say, within the first $200 \mathrm{~km}$ above the continuum forming layer, there would be space for only one tube with $R=100 \mathrm{~km}$. At most two such tubes might be stacked, since the entire photosphere has only a depth of some $400 \mathrm{~km}$. Also, it is clear from observations that the major portion of the magnetic flux is in the form of fields that is inclined with respect to the horizontal. The horizontal portion of the magnetic flux in which the (Evershed) flow is present should therefore only fill a part of the photospheric volume. Hence, geometrical considerations argue against option (1).

\subsection{Discussion}

Within the framework of option (2) our considerations suggest that the upflows are capable of heating the entire penumbra, if the horizontal flux tubes submerge below the photosphere within the length $l$. In this scenario the horizontal portions of the flux tubes lying at the solar surface are the tops of $\Omega$-like loops. These horizontal portions extend to, say,
$1000 \mathrm{~km}$ assuming a penumbral filament with a width of approximately $200 \mathrm{~km}$. This suggests that flows within magnetic flux tubes emerge as hot upflows and submerge as cool downflows throughout the penumbra. Such a scenario is consistent with the observation that the Evershed flow has a downward component not only at the outer edge, as found by Westendorp Plaza et al. (1997), but already well within the penumbra (Schlichenmaier \& Schmidt 2000; Schmidt \& Schlichenmaier 2000; del Toro Iniesta et al. 2001; Bellot Rubio et al. 2003).

Investigations of the evolution of thin flux tubes within the penumbra also provide evidence that downflow arches within the penumbra might be present (Jahn et al. 1996; Schlichenmaier 2002). These simulations suggest that tubes might have the shape of a sea serpent in the photosphere. The hot upflow along the tube cools in the photosphere as it flows outward. Later the gas follows the field back beneath the photosphere, where it heats up again and reappears in the photosphere as a hot upflow channel. Based on these results, one might speculate that one single tube is capable of forming two hot upflow channels, although there are still considerable uncertainties surrounding the properties of the second upflowing footpoint. The amount of heat that can be deposited in the penumbral photosphere in the second footpoint will depend on the diameter of the tube, and on the flow velocity. A radiatively cooled flow that re-enters the subphotosphere with a flow speed of, say, $10 \mathrm{~km} \mathrm{~s}^{-1}$, is effectively heated as long as the photon mean-free path is comparable to the diameter of the flux tube. This condition is fulfilled only in a depth range of roughly $100 \mathrm{~km}$ around the base of the photosphere, where it dwells for at least $10 \mathrm{~s}$. This lower limit corresponds to a vertically oriented flux tube. Since we do not expect the tube to be substantially inclined to the horizontal, the "dwelling" time should be significantly larger than $10 \mathrm{~s}$. Based on results from SBS1999, where a $12000 \mathrm{~K}$ hot tube with a diameter of $100 \mathrm{~km}$, embedded in an optically thin environment cools within $9 \mathrm{~s}$ to the ambient penumbral temperature, we expect that such an optically thin downflow would be fully heated up to the surrounding hot temperatures in the subphotosphere. We plan to carry out radiative transfer calculation to elaborate on this point in more detail. The latter argument indicates that one steady flow along a flux tube can in principle provide two or more hot upflow channels to carry sufficient energy to the penumbra.

\subsubsection{Relation to granulation}

In the steady flow scenario the penumbra is heated by a steady convective flow, qualitatively similar to the granulation (except that the former is guided by the magnetic field of the flux tube). One major difference to the quiet Sun lies in the geometry. In the quiet Sun the hot gas being transported to the surface by granulation easily carries sufficient energy to produce $F_{\odot} \simeq 1.35 F_{\text {pen }}$, even for a small upflow velocity (typically $1 \mathrm{~km} \mathrm{~s}^{-1}$ for granules). The salient feature of granular upflows is that they cover approximately half of the total area. They thus need to transport an excess energy flux corresponding to only $2 F_{\odot}$. In the penumbra, the upflow is channeled by magnetic flux tubes, and its velocity is considerably higher. 
We estimate that an upflow with a velocity of $4 \mathrm{~km} \mathrm{~s}^{-1}$ can deposit $F_{\text {upflow }}=8 F_{\text {sb }}$ (cf. Eqs. (3), (4)), i.e., 8 times the heat that is needed to account for the surplus brightness of the penumbra. Yet, the penumbral area which can be heated by such an upflow is limited. Therefore we argue for a return-flux within the penumbra, and thus restrict the length $l$ over which a particular upflow deposits its heat, and propose that the flow and its associated flux tube dives beneath the photosphere after the length $l$. For the standard parameters that we used in the estimates, this would mean that $R^{2} \pi / A \approx F_{\mathrm{sb}} / F_{\text {upflow }} \approx 13 \%$ of the surface area are covered by upflows. If some of these upflows are footpoints of re-appearing flux tubes (second or third footpoints in the picture of the sea serpent) which might be somewhat cooler, then this value would have to be correspondingly larger.

\subsubsection{Other heating mechanisms}

So far we have only considered magneto-convective heating processes for the penumbra. The scenario that we propose above features two key ingredients: (a) Magnetic fields with inclinations that vary on small spatial scales (being uncombed) and (b) flows channelled by the magnetic field while being embedded in a background at rest. In such a situation, it is conceivable that other heating sources are relevant for the penumbra: (a) ohmic dissipation of the electric currents at the boundary between the individual flux tube and the differently inclined magnetic field of the surroundings, and (b) dissipation occuring in an instability that is triggered by the strong shear flow between the flow channel and the surroundings. Detailed investigations of such mechanisms are beyond the scope of the present paper and need to be considered in future work. Here, we restrict ourselves to a crude estimate of an upper limit of the dissipated energy which could contribute to the brightness of the penumbra.

To give an estimate for the upper limit of magnetic dissipation in the penumbra, we consider a horizontal flux tube with $R=100 \mathrm{~km}$, and a magnetic field strength, $B=1500$ Gauss. For an upper limit, we assume that the currents that form such a flux tube are fully converted into heat by ohmic dissipation, such that the released energy per unit area is given by the magnetic energy of the tube per unit area (cf. Sect. 2.2):

$E_{\text {magnetic }}=\frac{R^{2} \pi l}{2 R l} \cdot \frac{B^{2}}{8 \pi}=\frac{R \pi}{2} \cdot \frac{B^{2}}{8 \pi}$.

Since the magnetic pattern within a sunspot does not change within $80 \mathrm{~min}$ (cf. Paper I), it seems reasonable to assume that it takes at least $80 \mathrm{~min}$ before the flux tube is fully dissipated by ohmic diffusion. Therefore, the energy flux is

$F_{\text {magnetic }}=\frac{E_{\text {magnetic }}}{80 \mathrm{~min}} \approx 3 \times 10^{8} \frac{\mathrm{ergs}}{\mathrm{s} \mathrm{cm}^{2}}$.

This value is 100 times smaller than the surplus brightness of the penumbra (cf. Eq. (3)). Hence it seems that dissipation of magnetic energy does not play a significant role in heating (brightening) the penumbra. Scharmer et al. (2002) come to a similar conclusion.
An upper limit for the dissipated heat due to shear flow instabilities can be given by assuming that all the kinetic energy of the flow is dissipated. We use the typical values that we consider reasonable for a penumbral flux tube carrying the Evershed flow: $R=100 \mathrm{~km}, \rho=2 \times 10^{-7} \mathrm{~g} \mathrm{~cm}^{-3}$, and $v=10 \mathrm{~km} \mathrm{~s}^{-1}$. One may expect that the released heat is distributed over a rectangular area along the tube which has a length of $l=1000 \mathrm{~km}$ and a width of $2 R$. Then the heat flux due to dissipation of kinetic energy is

$F_{\text {kinetic }}=\rho \frac{v^{2}}{2} \cdot \frac{\left(R^{2} \pi\right) v}{2 R \cdot l} \approx 1.5 \times 10^{10} \frac{\mathrm{ergs}}{\mathrm{s} \mathrm{cm}^{2}}$,

which is the same order of magnitude as the surplus brightness of the penumbra. Therefore, this process can, in principle, supply a significant fraction of the penumbral brightness, and should be studied in detail in future work.

\section{Conclusions}

The best known convective phenomenon on the Sun is granulation. In the case of granulation, energy can be transported extremely efficiently in a quasi-steady state (overturning convection). For energy transport within sunspots, interchange convection has been proposed. Within this framework, one expects intensity variations in time, due to rising hot and sinking cool flux tubes (Jahn \& Schmidt 1994). Here we have compared estimates with observations to gain some insight into such time-dependence in sunspots.

The observed small-scale structures have lifetimes ranging from $30 \mathrm{~min}$ (intensity structures in the outer penumbra) to well over two hours (magnetic features). Also, the lifetime of the magnetic pattern is greater than that of the intensity pattern (cf. Paper I). These long lifetimes pose a problem for interchange convection as a mechanism to transport heat: First, we find that the radiative cooling time of hot plasma in a cool photosphere is an order of magnitude shorter than the intensity variations seen in penumbrae, and second, the time scale at which the magnetic configuration, due to interchanging flux tubes, should change in order to transport the proper amount of energy, is also much smaller than observed.

In view of this result we have explored a further avenue, namely the transport of energy by means of a steady upflow along flux tubes, i.e., heating through the enthalpy flux associated with the Evershed flow. The presence of such an enthalpy flux is predicted by the simulations of Schlichenmaier et al. (1998a,b).

We estimate that for typical parameters such upflow channels supply enough energy to account for the surplus brightness of the penumbra with respect to the umbra. However, these upflow channels can only supply energy for a limited area. To heat the entire width of a penumbra along a radial section, several upflow channels are needed. Since only a limited number of flux tubes can be stacked in the photosphere, the tubes have to submerge within 1000-2000 km after their footpoint, i.e., they cannot continue to be horizontal $1000-2000 \mathrm{~km}$ after the footpoint as suggested in the simulations mentioned above. Then, this mechanism could indeed supply a sufficiently large 
enthalpy flux to heat the penumbra. Support for this scenario is given by observation of downflows within the penumbra and by simulations of the moving tube model, which demonstrate that the tube can in principle have the shape of a sea serpent with hot up- and cool downflows (cf. Sect. 3.1).

Thus, based on observational constraints and theoretical estimates, we conclude that interchange convection as the dominant energy transport mechanism within the penumbra can be ruled out. Instead, we propose that hot upflows and cool downflows as in solar granulation could provide the necessary enthalpy flux. As suggested by numerical simulations, these flows occur in magnetic flux tubes: The upflow is driven by the superadiabaticity of the subphotospheric stratification. The hot plasma cools radiatively in the photosphere and might submerge in downflow channels, as is suggested by observations.

Acknowledgements. We are grateful to O. Steiner and I. Rüedi for many fruitful discussions and suggestions. We thank them and L. Bellot Rubio for useful comments on the manuscript.

\section{References}

Bellot Rubio, L. R., Balthasar, H., Collados, M., \& Schlichenmaier, R. 2003, A\&A, 403, L47

Danielson, R. E. 1961, ApJ, 134, 289

Grossmann-Doerth, U., \& Schmidt, W. 1981, A\&A, 95, 366

Hurlburt, N. E., Matthews, P. C., \& Proctor, M. R. E. 1996, ApJ, 457, 933

Hurlburt, N. E., Matthews, P. C., \& Rucklidge, A. M. 2000, Sol. Phys., 192, 109

Jahn, K. 1989, A\&A, 222, 264

Jahn, K. 1997, in 1st Advances in Solar Physics Euroconference. Advances in Physics of Sunspots, ed. B. Schmieder, J. C. del Toro Iniesta, \& M. Vazquez, ASP Conf. Ser., 118, 122

Jahn, K., Schlichenmaier, R., \& Schmidt, H. U. 1996, Astr. Lett. Commun., 34, 59

Jahn, K., \& Schmidt, H. U. 1994, A\&A, 290, 295

Maltby, P. 1964, Astrophys. Norv., 8, 205

Pizzo, V. J. 1986, ApJ, 302, 785

Pizzo, V. J. 1990, ApJ, 365, 764
Pizzo, V. J., MacGregor, K. B., \& Kunasz, P. B. 1993, ApJ, 413, 764

Rimmele, T. R. 1995, A\&A, 298, 260

Scharmer, G. B., Gudiksen, B. V., Kiselman, D., Löfdahl, M. G., \& Rouppe van der Voort, L. H. M. 2002, Nature, 420, 151

Schlichenmaier, R. 1999, in High Resolution Solar Physics: Theory, Observations, and Techniques, ed. T. R. Rimmele, K. S. Balasubramaniam, \& R. R. Radick, ASP Conf. Ser., 183, 91

Schlichenmaier, R. 2002, Astron. Nachr., 323, 303

Schlichenmaier, R., Bruls, J. H. M. J., \& Schüssler, M. 1999, A\&A, 349,961

Schlichenmaier, R., \& Collados, M. 2002, A\&A, 381, 668

Schlichenmaier, R., Jahn, K., \& Schmidt, H. U. 1998a, ApJ, 493, L121

Schlichenmaier, R., Jahn, K., \& Schmidt, H. U. 1998b, A\&A, 337, 897

Schlichenmaier, R., \& Schmidt, W. 2000, A\&A, 358, 1122

Schmidt, H. U. 1991, Geophys. Astrophys. Fluid Dyn., 62, 249

Schmidt, W., \& Schlichenmaier, R. 2000, A\&A, 364, 829

Sobotka, M. 1997, in 1st Advances in Solar Physics Euroconference. Advances in Physics of Sunspots, ed. B. Schmieder, J. C. del Toro Iniesta, \& M. Vazquez, ASP Conf. Ser., 118, 155

Sobotka, M., \& Sütterlin, P. 2001, A\&A, 380, 714

Solanki, S. 2001, in Encyclopedia of Astronomy and Astrophysics (Nature Publishing Group), 3180

Solanki, S. K. 2003, A\&ARv, 11, 153

Solanki, S. K., \& Rüiedi, I. 2003, A\&A, 411, 249

Solanki, S. K., \& Schmidt, H. U. 1993, A\&A, 267, 287

Stanchfield, D. C. H., Thomas, J. H., \& Lites, B. W. 1997, ApJ, 477, 485

Stix, M. 2002, The Sun. An Introduction (Berlin, Heidelberg: Springer-Verlag), 2nd ed.

Sütterlin, P. 2001, A\&A, 374, L21

Thomas, J. H., \& Weiss, N. O. 1992, in NATO ASIC Proc. 375: Sunspots. Theory and Observations, ed. J. H. Thomas, \& N. O. Weiss, 3

del Toro Iniesta, J. C., Bellot Rubio, L. R., \& Collados, M. 2001, ApJ, 549, L139

Weiss, N. O. 1997, in 1st Advances in Solar Physics Euroconference. Advances in Physics of Sunspots, ed. B. Schmieder, J. C. del Toro Iniesta, \& M. Vazquez, ASP Conf. Ser., 118, 21

Weiss, N. O. 2002, Astron. Nachr., 323, 371

Westendorp Plaza, C., del Toro Iniesta, J. C., Ruiz Cobo, B., et al. 1997, Nature, 389, 47 